\title{
Identification of NuoX and NuoY Ligand Binding Specificity in the Campylobacter Jejuni Complex I
}

Lirio I. Calderon-Gomez ${ }^{1}$, Christopher J. Day ${ }^{1}$, Lauren E. Hartley-Tassell ${ }^{1}$, Jennifer C. Wilson ${ }^{1}$, George L. Mendz ${ }^{2}$ and Victoria Korolik $^{1^{\star}}$

${ }^{1}$ Institute for Glycomics, Griffith University, Gold Coast, QLD, Australia

${ }^{2}$ School of Medicine, The University of Notre-Dame Australia, NSW, Sydney, Australia

"Corresponding author: Professor Victoria Korolik, Institute for Glycomics, Griffith University, Gold Coast, Queensland, 4222, Australia, Tel: +61755528321; E-mail: v.korolik@griffith.edu.au

Received date: April 05, 2017; Accepted date: April 21, 2017; Published date: April 26, 2017

Copyright: @2017 Calderon-Gomez LI, et al. This is an open-access article distributed under the terms of the Creative Commons Attribution License, which permits unrestricted use, distribution, and reproduction in any medium, provided the original author and source are credited.

\begin{abstract}
The components of the proton pump NADH:ubiquinone (Complex I) of the respiration pathway have been identified in the $C$. jejuni genome. However, the paradigm genes nuoE and nuoF encoding subunits of the NADH dehydrogenase module of Complex I are absent. Instead the genes cj1575c and cj1574c encoding NuoX and NuoY are present in the loci corresponding to nuoE and nuoF, respectively. Bioinformatics analyses showed the presence of nuoX and nuoY homologues in all sequenced strains of $C$. jejuni and in other Campylobacter species, as well as the presence of orthologues in other $\varepsilon$-Proteobacteria.
\end{abstract}

To understand the involvement of the NuoX and NuoY proteins in the respiration of $C$. jejuni and to characterize their ligand binding specificity and affinity, a tricarboxylic acid cycle array was developed as a tool to identify proteins that can bind to intermediates of this cycle as well as other metabolites. This array showed that NuoX bound FAD ${ }^{2+}$, and NuoY bound $\mathrm{FAD}^{2+}$ and the electron donors malate and lactate. Saturation Transfer Difference Nuclear Magnetic Resonance studies confirmed the NuoY binding ligands, and suggested that the flavin moiety of FAD ${ }^{2+}$ interacted more strongly with NuoY than the adenine moiety. Affinity data generated by Surface Plasmon Resonance indicated that NuoY bound to $F A D^{2+}$ with a $K_{\mathrm{D}}$ of $337 \mathrm{nM}$; NuoX and NuoY had an affinity for NADH of a $K_{\mathrm{D}}$ of $403 \mathrm{nM}$ and $478 \mathrm{nM}$, respectively, and a ten-fold lower affinity for both $\mathrm{NAD}^{+}$and $\mathrm{FAD}^{2+}$. The data suggested that the flavin-adenine dinucletoide could be bound preferentially to the NAD in the Complex I of $C$. jejuni.

Keywords: Complex I; NuoX; NuoY; Campylobacter jejuni; Ligand binding

\section{Introduction}

The major foodborne intestinal pathogen Campylobacter jejuni is an obligate microaerophile and consequently requires oxygen for growth. Oxygen is utilized as a terminal electron acceptor in its respiratory chain, but the bacterium cannot grow under fully aerobic conditions or grows very poorly [1]. In addition, the genome of $C$. jejuni encodes a branched electron transport system, which allows for utilization of multiple electron donors including organic acids, as well as various electron acceptors [2], including some metabolites of the tricarboxylic acid (TCA) cycle [3-6].

Complex I (EC 1.6.5.3) is the NADH:ubiquinone oxidoreductase enzyme of the aerobic respiratory chain widely found in bacteria and archaea, as well as in the mitochondria of eukaryotes [7]. It has been well characterized in Escherichia coli [8] and Paracoccus denitrificans [9]. It performs the first enzymatic step in the respiratory chain by catalyzing the transfer of electrons from $\mathrm{NADH}$ to a quinone pool coupled with the translocation of protons across the membrane [10].

In bacteria, Complex I is a large ensemble of proteins encoded by 14 different genes arranged in an operon designated nuo (NADH:ubiquinone oxidoreductase) with a highly conserved order [11]. The complex quaternary structure is L-shaped [9,12,13]; the membrane-embedded long segment of the ' $\mathrm{L}$ ' is the P-module consisting of seven hydrophobic proteins called subunits Nuo H, K, J,
A, N, M, and L [14]. The short arm protruding out of the membrane into the cytoplasm contains all the identified prosthetic groups of Complex I except quinones. The seven proteins (NuoB-G and NuoI) are peripheral subunits and make up two modules: (1) the N-module which is a NADH-oxidizing unit, composed of subunits Nuo E, F and $\mathrm{G}$ in bacterial enzymes, and (2) a Q-module constituted by NuoB, NuoC, NuoD and NuoI, that connects the N-module of the peripheral fragment to the P-module of the membrane fragment [15]. Subunit NuoE possesses a binuclear Fe-S center and is most likely involved in binding NADH $[8,16]$. Subunit NuoF has a catalytic NADH binding site, a noncovalently bound flavin mononucleotide (FMN), and an iron-sulfur (Fe-S) center [17-19]. Subunits NuoE and NuoF along with subunit NuoG, in some cases, can be dissociated from the rest of the complex to form a soluble $\mathrm{N}$-module with $\mathrm{NADH}$ dehydrogenase activity [17-19].

In the $C$. jejuni Complex I operon, nuoE and nuoF are absent; instead, the genes $c j 1575 c$ and $c j 1574 c$ are present and designated nuo $X$ and nuoY $[2,20]$. The remaining subunits in the $C$. jejuni Complex I have been identified through sequence similarities to the proteins found in other bacteria such as E. coli [8] and P. denitrificans [9]. Nuo operon forms one of two structured operons in the $C$. jejuni NCTC 11168 genome, the other being a well characterized lipoologosaccharide biosynthesis locus [2]. It is interesting to note that, despite the fact that operons are not common in $C$. jejuni, the Nuo operon structure in $C$. jejuni is maintained and reflects that found in other bacteria with genes encoding NuoX and NuoY replacing those encoding NuoE and NuoF. The two proteins NuoX and NuoY have 
shorter amino acid residue sequences compared to the NuoE and NuoF proteins of other species, and have very low sequence similarity to other known Nuo proteins $[8,20]$. Hoffman [21] demonstrated that in $C$. jejuni, $\mathrm{NADH}$ is a poor electron donor to Complex I. Previous studies proposed that NuoX and NuoY act as electron acceptors from flavodoxin dinucleotide rather than the more common donor NADH [22]. Thus, it was a unexpected finding that the other components of the proton pump NADH:ubiquinone pathway were identified in the $C$. jejuni genome [2].

The genome of $C$. jejuni encodes two other enzymes, pyruvate ferredoxin oxidoreductase (PFOR) and 2-oxoglutarate oxidoreductase (OOR), which use ferredoxin or flavodoxin as electron acceptor in the TCA cycle; specifically, for the formation of acetyl-CoA by a coenzyme A-dependent oxidative decarboxylation of pyruvate [23,24].

The bacterium has the capacity to operate a complete oxidative TCA cycle, with all of the required enzymes present [2]. This includes homologues of the $\alpha$ - and $\beta$-subunits of succinyl-CoA synthetase and a NAD-linked malate dehydrogenase. $C$. jejuni encodes a dualfunctioning fumarate reductase, that also has a succinate:quinone reductase activity, which is consistent with the presence of an oxidative TCA cycle $[25,26]$.

The unique Complex I of $C$. jejuni aerobic respiratory chain lacking the NuoE and NuoF subunits which bind NADH in other bacteria, suggests that in this bacterium the actual source of electrons for Complex I may not be reduced NAD $[21,25,27]$. The ability of $C$. jejuni to express fumarate reductase may allow anaerobic electron transport using fumarate as the terminal electron acceptor [25]. More recently, it has been shown that a dihaemcytochrome $c$ thiosulphate dehydrogenase of $C$. jejuni can use tetrathionate and thiosulphate for energy metabolism [28].

Potential interactions between NuoX and NuoY were investigated employing a yeast two-hybrid interaction system. This served to ascertain whether the arrangement of the genes nuo $X$ and nuo $Y$ in the $C$. jejuni genome encoded proteins that interacted.

Traditional substrate utilization studies provided information on putative substrates for the $C$. jejuni electron transport chain and TCA cycle [21,27]. However, while these studies identified substrates that are metabolized by $C$. jejuni, they did not provide information regarding the binding partners or the nature of the interactions.

To address these issues we have developed novel methodology, a TCA array, that uses the intermediates $\mathrm{NAD}^{+}$, succinate, malate, fumarate, acetyl-CoA, $\alpha$-ketoglutarate and flavin adenine dinucleotide $\left(\mathrm{FAD}^{2+}\right.$ ), in order to understand the role of these substrates in $C$. jejuni metabolism, and to characterize potential protein-binding partners of the respiratory proteins NuoX and NuoY. Furthermore, Saturation Transfer Difference Nuclear Magnetic Resonance Spectroscopy (STDNMR) and Surface Plasmon Resonance (SPR) were used to detect and characterize protein-ligand binding, and to confirm interactions between binding partners.

\section{Materials and Methods}

\section{Microbial strains, growth conditions, plasmids and primers}

The $C$. jejuni strains used in this study were the original clinical Skirrow's isolate NCTC11168-O (hereafter Cj11168) kindly provided by D. G. Newell (VLA, London, UK) [2], and C. jejuni strain 81116 from our laboratory's collection. The bacteria were grown on Columbia agar supplemented with 5\% defibrinated horse blood (HBA) with Skirrow antibiotic supplement (Oxoid, Thermo Fisher Scientific, Adelaide, SA, Australia); the plates were grown overnight at $42^{\circ} \mathrm{C}$ under microaerobic conditions. The host E. coli strains DH5a and BL21 (DE3) were grown on Luria-Bertani media (Oxoid). Host yeast strains were grown and prepared according to the manufacturer's instructions (Clontech Laboratories Inc., Mountain View, CA, USA).

Primers were designed for the nuoX (cj1575c) and nuoY (cj1574c) genes based on the nucleotide sequence of $C$. jejuni strain NCTC11168-GS [2] and used with $C$. jejuni strain 81116. Oligonucleotide primers were synthesized by Invitrogen (Invitrogen Australia Pty. Ltd., Mount Waverley, VIC, Australia) and are listed in Table S1.

\section{Yeast two-hybrid interaction system}

Yeast two-hybrid analyses of protein-protein interactions were performed using the Matchmaker GAL4 Two-Hybrid System 3 (Clontech). DNA sequences encoding the $C$. jejuni NCTC11168 nuoX and nuoY were subcloned into both pGADT7 and pGBKT7 subsequent to initial cloning into pGEM-T Easy.

Reciprocal plasmid construct combinations (Table S2) were tested in the yeast host strain AH109. Co-transformants were grown initially on low stringency media (SD/-Leu/-Trp), and were streaked subsequently onto intermediate stringency media (SD/-His/-Leu/-Trp) and high stringency (SD/-Ade/-His/-Leu/-Trp) media. Growth of yeast co-transformants was examined after 5-7 days. The absence of growth indicated no protein-protein interactions, whereas growth of yeast cotransformants on intermediate stringency and/or high stringency indicated protein-protein interactions. The assays of positive and negative controls consisted of interactions of pGADT7-T with pGBKT7-53 or pGBKT7-Lam (Clontech). All control cotransformants were included on each protein-protein interaction assay plate, and each assay was performed at least twice to verify the results. Autonomous activation of reporter gene expression was assayed using AH109 co-transformed with each recombinant plasmid in combination with the reciprocal vector.

\section{Expression of the NuoX and NuoY proteins}

Expression of NuoX and NuoY was performed by cloning the coding regions of nuoX or nuoY genes into the pET-19b expression system in frame with an $N$-terminal polyhistidine tag; the proteins were expressed as previously described [29]. Recombinant protein expression was induced using $1 \mathrm{mM}$ IPTG when the $\mathrm{OD}_{600}$ of the culture reached 0.8-1.0, and expression of the His-NuoX or His-NuoY fusion proteins was verified by SDS-PAGE and Western Blot analyses. The solubilities of the His-NuoX and His-NuoY fusion proteins were confirmed as described previously [30]. Purification of recombinant proteins was performed using the His-Select ${ }^{\circ}$ Nickel Affinity Gel resin according to the manufacturer's protocols.

\section{TCA arrays}

Tricarboxylic acid (TCA) cycle intermediates and other metabolites (Table 1) were dissolved in water and spotted onto epoxy functionalised glass slides (Arrayit Corporation, Sunnyvale, CA, USA) using a non-contact Piezorray Microarray Printer (Perkin Elmer Life and Analytical Sciences, Downers Grove, IL, USA), as previously described [31], with the appropriate modifications [29]. The array included eleven different compounds of which seven are directly 
involved in the TCA cycle [26], including oxidised $a-\mathrm{NAD}^{+}, \alpha-$ ketoglutarate, acetyl coenzyme-A, oxidised flavin adenine dinucleotide $\left(\mathrm{FAD}^{2+}\right)$, sodium fumarate, sodium succinate, and sodium malate; and three that are not directly involved in the TCA cycle: L-lactic acid, sodium sulfide and bile salts (Table 1).

\begin{tabular}{|c|c|}
\hline \multicolumn{2}{|c|}{ Substrates used for TCA array assay } \\
\hline Code & Substrate \\
\hline $1 \mathrm{~A}$ & $\mathrm{NAD}^{+} 50 \mathrm{mM}$ \\
\hline 1B & Succinate $50 \mathrm{mM}$ \\
\hline $1 \mathrm{C}$ & Sodium L-lactate $50 \mathrm{mM}$ \\
\hline 1D & Sodium sulfide $50 \mathrm{mM}$ \\
\hline $1 \mathrm{E}$ & Sodium fumarate $50 \mathrm{mM}$ \\
\hline $1 \mathrm{~F}$ & Malic acid $50 \mathrm{mM}$ \\
\hline $1 \mathrm{G}$ & Malic acid sodium salt $50 \mathrm{mM}$ \\
\hline $1 \mathrm{H}$ & a-ketoglutarate $50 \mathrm{mM}$ \\
\hline $2 \mathrm{~A}$ & $\mathrm{NAD}^{+} 10 \mathrm{mM}$ \\
\hline $2 \mathrm{~B}$ & Succinate $10 \mathrm{mM}$ \\
\hline $2 \mathrm{C}$ & Sodium L-lactate $10 \mathrm{mM}$ \\
\hline $2 \mathrm{D}$ & Sodium sulfide $10 \mathrm{mM}$ \\
\hline $2 \mathrm{E}$ & Sodium fumarate $10 \mathrm{mM}$ \\
\hline $2 \mathrm{~F}$ & Malic acid $10 \mathrm{mM}$ \\
\hline $2 \mathrm{G}$ & Malic acid sodium salt $10 \mathrm{mM}$ \\
\hline $2 \mathrm{H}$ & a-ketoglutarate $10 \mathrm{mM}$ \\
\hline $3 \mathrm{~A}$ & $\mathrm{FAD}^{2+} 10 \mathrm{mM}$ \\
\hline $3 B$ & Bile Salts $10 \mathrm{mg} / \mathrm{ml}$ \\
\hline $3 C$ & Acetyl-CoA $10 \mathrm{mM}$ \\
\hline 4B & Bile Salts $100 \mathrm{mg} / \mathrm{ml}$ \\
\hline
\end{tabular}

Table 1: Substrates used in TCA array for all assays.

The metabolites were printed in spots of 0.3-0.6 nl onto epoxide activated glass array substrates to produce the TCA array [31]. The compounds were spotted at concentrations of $10-50 \mathrm{mM}$, then neutralised as per the manufacturer's instructions and the arrays stored at $4^{\circ} \mathrm{C}$. Verification of TCA arrays was performed using human glutathione reductase and a glutathione reductase antibody (SigmaAldrich, Castle Hill, NSW, Australia). Glutathione reductase is involved in oxidative stress responses and is produced widely by plants, animals, bacteria and yeast; only the trypanosome protozoa and drosophila flies do not encode a glutathione reductase enzyme [32,33], instead a functional analogue is present in both these organisms. Glutathione reductase utilizes NADPH and $\mathrm{FADH}_{2}$ to catalyze the reduction of glutathione disulfide to glutathione sulfhydryl, which can then act as an antioxidant in the cell. This protein was chosen to verify TCA array interactions by showing that the surface bound ligands can properly interact with soluble proteins.
Binding to TCA arrays was deemed significant if the average relative fluorescence unit (RFU) of each spotted compound was greater than the mean background. The mean background was defined as the average RFU of empty spots plus three standard deviations, and statistical analyses showed a $p$ value of $<0.0001$.

For array measurements, NuoX and NuoY purified proteins were suspended in PBS with $2 \mathrm{mM} \mathrm{MgCl} / \mathrm{CaCl}_{2}$ and pre-complexed with a primary mouse anti-His and AlexaFluor488 secondary and tertiary antibodies (Invitrogen). Proteins were hybridized to the array for 15 minutes at $37^{\circ} \mathrm{C}$ in the dark, washed in warm PBS with BSA and in PBS with Tween-200.05\% (v/v) for 15 min each, and had a final rinse in PBS. Slides were dried scanned by a ProScanArray Microarray 4laser scanner (Perkin Elmer; Waltham, Massachusetts, USA) using a Blue Argon 488 excitation laser (excitation $495 \mathrm{~nm}$, emission $529 \mathrm{~nm}$ ). Image analysis was carried out using the ProScanArray imaging software, ScanArray Express (Perking Elmer).

\section{Saturation transfer difference NMR spectroscopy}

Purified proteins were concentrated and prepared in $50 \mathrm{mM}$ deuterated sodium acetate $-\mathrm{d}_{3}$ buffer, $\mathrm{pH}$ 6.5. The addition of ligand $\left(10 \mathrm{mM}\right.$ in $\left.2 \mathrm{H}_{2} \mathrm{O}\right)$ to His-NuoY, gave a His-NuoY:ligand ratio of 1:200 for all compounds in a total volume of $700 \mu$ l. The ligands investigated included $\alpha-\mathrm{NAD}^{+}$, flavin adenine dinucleotide $\left(\mathrm{FAD}^{2+}\right)$, sodium fumarate, L-lactate and L-(-)malate. Proton $(1 \mathrm{H})$ NMR experiments were performed at $288 \mathrm{~K}$ on a Bruker Avance $600 \mathrm{MHz}$ spectrometer equipped with a $5 \mathrm{~mm} \mathrm{TCl}$ cryoprobe with $\mathrm{Z}$-axis gradients. Spectra were acquired with $1 \mathrm{~K}$ scans, and a relaxation delay at least $>5$ times the $T_{1}$ value of the longest relaxation time of protons in the ligand being studied. Proton $\mathrm{T}_{1}$ values were determined using the inversion recovery method.

For the STD-NMR experiments the protein was saturated with an on-resonance at a frequency of $-600 \mathrm{~Hz}$ in the aliphatic region of the spectrum, and an off-resonance at $20,000 \mathrm{~Hz}$ with a cascade of 40 selective Gaussian shaped pulses of $50 \mathrm{~ms}$ with a $100 \mu$ s delay between each pulse, resulting in a total saturation time of 2 seconds. Difference STD-NMR spectra were obtained by superimposing on resonance and off-resonance spectra followed by a subtraction to obtain a difference spectrum containing the STD signals of binding ligands. As controls, ligand only spectra were acquired, and no signal was observed in their difference spectra.

\section{Surface plasmon resonance studies}

Binding of various compounds to His-Nuo purified proteins were assessed using a Biacore T100 instrument (GE Healthcare Lifesciences, Rydalmere, NSW, Australia). For all experiments, Ni-NTA series 2 sensor chips (GE Healthcare Lifesciences) were used according to the manufacturer's instructions. The NTA sensor chip surface was stripped with $0.1 \mathrm{M}$ EDTA, and charged with $500 \mu \mathrm{M} \mathrm{NiCl}_{2}$ solution; the Histagged proteins were adjusted to $100 \mu \mathrm{g} / \mathrm{ml}$ in running buffer $(0.01 \mathrm{M}$ HEPES, $0.15 \mathrm{NaCl}, 50 \mu \mathrm{M}$ EDTA, $0.05 \%$ Tween 20, pH 7.4), and injected over the NTA chip at $10 \mu \mathrm{l} / \mathrm{min}$ until the desired saturation was reached. Ligand-analyte interactions were detected using multicycle kinetics assay employing concentrations of analyte ranging from $0.5 \mathrm{nM}$ to $10 \mathrm{mM}$. The sensorgram data for each of the methods used were analyzed using the BIAevaluation software version 2.0 (Biacore, GE Healthcare Lifesciences). 


\section{Statistical analyses}

Statistical analyses were performed using student's t-tests and oneway ANOVA tests employing Microsoft Excel or Prism software. All statistics were performed with an alpha value of 0.05 .

\section{Results}

\section{In silico analyses}

Comparative bioinformatics analyses of the predicted amino acid residue sequences $C$. jejuni NCTC11168 genes cj1575c (nuoX) and cj1574c (nuoY) were performed using Blastp and the Conserved Domain Search tools as described by Marchler and Bryant [34]. Homologues of the genes nuoX and nuoY were identified in all sequenced strains of $C$. jejuni to date, with predicted amino acid residue identities greater than $99 \%$. Orthologues of these genes were present in other Campylobacter species with greater than 50\% predicted amino acid residue similarities. Analyses of the nucleotide sequences encoding $C j 1575 c$ and $C j 1574 c$, did not yield specific conserved domains in the amino acid residue sequences of either $C$. jejuni strains or related species. No significant similarities were found in the $C$. jejuni genome to the proteins encoded by the $n u o E$ or nuo $F$ genes of E. coli strains.

In silico analyses showed that NuoX and NuoY protein orthologues were encoded also in the genomes of other genera of $\varepsilon$-Proteobacteria, such as Helicobacter, Wollinella and Arcobacter with approximately $31-55 \%$ amino acid residue similarities to the NuoX or NuoY proteins in $C$. jejuni. Comparative analyses identified also the presence of genes similar to nuoX and nuoY in other bacteria such as Sulfuricurvum spp., Sulfuromonas spp. and Nitratiruptor spp., with between $32 \%$ and $64 \%$ predicted amino acid residue similarities to the NuoX residue sequence, and up to $31 \%$ similarity to the NuoY sequence.

\section{Analysis of protein-protein interactions between the $C$. jejuni NCTC11168 Nuo proteins}

A yeast-2-hybrid protein interaction system was used to investigate putative interactions of NuoX and NuoY proteins. The target vector expressing $C$. jejuni NCTC11168 NuoX and NuoY proteins fused to the GAL-4 AD was co-transformed with each of the recombinant plasmids expressing the GAL-4 DNA-BD fused to NuoX and NuoY proteins in reciprocal pair-wise combinations (Table S2). Strong interactions were detected between the NuoX and NuoY proteins (Table S3).

\section{TCA array development and verification using human glutathione reductase}

Considering that Complex I NuoE and NuoF subunits bind NADH in many bacteria, the binding of $C$. jejuni NuoX and NuoY to this dinucleotide and other metabolites, including intermediates of the TCA cycle was examined. To this end, a TCA array was developed, verified using glutathione reductase as a positive control, and employed to identify proteins that could bind to the various compounds in the array (Table 1). It was utilized to elucidate the binding specificities of NuoX or NuoY to metabolites in the array.

Glutathione reductase interacted with $\mathrm{NAD}^{+}$and $\mathrm{FAD}^{2+}$ on the array, in agreement with published studies [22]. This enzyme bound also to bile salts on the array. These bile salts are a mixed extract of salts of organic acids and other compounds found in bile. Thus, detection of interactions between glutathione reductase and bile salts provided evidence that compounds present in these salts were capable of binding the reductase. No other interactions were observed between glutathione reductase and the TCA substrates printed onto the array. These results with known ligands for glutathione reductase supported the use of this novel tool for identification of ligands to proteins. Also, it showed the capability of the array to reveal novel interactions such as those of the reductase with bile salts.

\section{Interactions between $C$. jejuni NuoX and NuoY proteins and various substrates}

Since salts of citrate, $a$-ketoglutarate, succinate, fumarate and malate, and bile salts are chemoattractants of $C$. jejuni [35], the interactions of purified His-NuoX and His-NuoY proteins with ligands using the TCA array were examined.

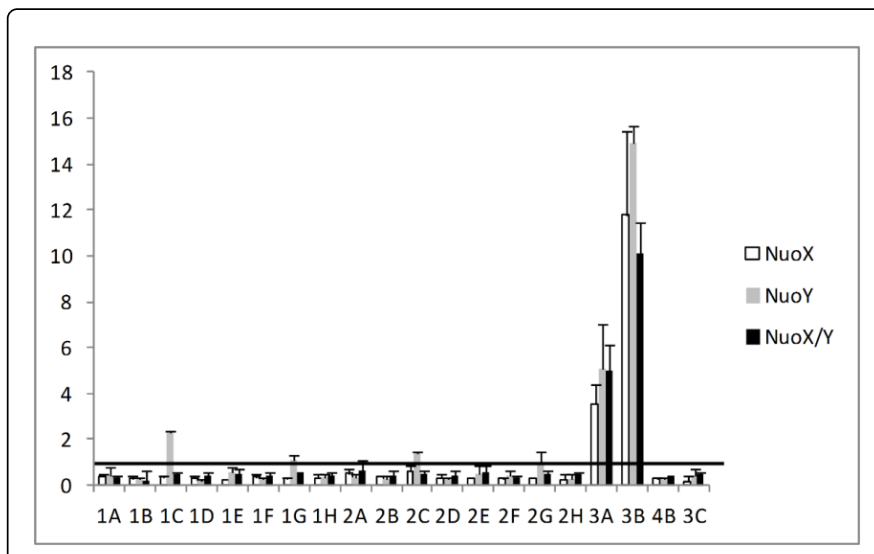

Figure 1: Comparison of the TCA array binding profiles showing His-NuoX (white), His-NuoY (grey) and His-NuoX/His-NuoY (black) with the substrates tested (Table 1).

NuoX bound to $\mathrm{FAD}^{2+}(3 \mathrm{~A})$ and bile salts (3B and $\left.4 \mathrm{~B}\right)$; NuoY bound to $\mathrm{FAD}^{2+}(3 \mathrm{~A})$, malate (1F and $\left.1 \mathrm{G}\right)$, lactate $(1 \mathrm{C}$ and $2 \mathrm{C})$ and bile salts (3B) (Figure 1 and Table 2). Counter-intuitively, when NuoX and NuoY proteins were mixed at concentrations of $100 \mu \mathrm{g} / \mathrm{ml}$ each to allow for potential formation of heterodimers, they bound only to $\mathrm{FAD}^{2+}(3 \mathrm{~A})$ and bile salts (3B and $\left.4 \mathrm{~B}\right)$ (Figure 1 and Table 2), but not to any of the other compounds. Interestingly, neither protein interacted with a-ketoglutarate, succinate or fumarate. Like glutathione reductase, NuoX and NuoY bound to $\mathrm{FAD}^{2+}$ and bile salts, suggesting the presence in these salts of compounds able to interact with reductases. Overall these results provided evidence of selective binding of proteins and indicated that $\mathrm{FAD}^{2+}(3 \mathrm{~A})$ was strongly recognised by both NuoX and NuoY as well as by the heterodimer formed by these proteins.

\section{STD-NMR spectroscopy of NuoY}

Saturation Transfer Difference ${ }^{1} \mathrm{H}-\mathrm{NMR}$ (STD-NMR) spectroscopy was employed to study further Nuo protein-ligand interactions. The STD-NMR spectra were obtained by subtracting the on-resonance and off-resonance spectra. As described above no STD-NMR signals were observed with His-NuoX were attempted with the His-NuoX protein $(11.3 \mathrm{kDa})$. An explanation could be that observation of STD-NMR spectra depends on the efficient spread of magnetization through spin- 
diffusion events which are not as efficient for a small size protein such as NuoX [36].

\begin{tabular}{|l|l|l|l|}
\hline Protein & Name & Code & Fold increase \\
\hline \multirow{3}{*}{ His-NuoX } & FAD $^{2+} 10 \mathrm{mM}$ & $3 \mathrm{~A}$ & $3.55 \pm 0.87$ \\
\cline { 2 - 4 } & Bile salts $10 \mathrm{mg} / \mathrm{ml}$ & $3 \mathrm{~B}$ & $11.77 \pm 3.65$ \\
\hline \multirow{3}{*}{ His-NuoY } & Sodium L-lactate $50 \mathrm{mM}$ & $1 \mathrm{C}$ & $2.27 \pm 0.11$ \\
\cline { 2 - 4 } & Malic acid sodium salt $50 \mathrm{mM}$ & $1 \mathrm{G}$ & $1.12 \pm 0.17$ \\
\cline { 2 - 4 } & Sodium L-lactate $10 \mathrm{mM}$ & $2 \mathrm{C}$ & $1.41 \pm 0.06$ \\
\cline { 2 - 4 } & Malic acid sodium salt $10 \mathrm{mM}$ & $2 \mathrm{G}$ & $0.96 \pm 0.54$ \\
\cline { 2 - 4 } & FAD ${ }^{2+} 10 \mathrm{mM}$ & $3 \mathrm{~A}$ & $5.08 \pm 1.98$ \\
\cline { 2 - 4 } & Bile salts $10 \mathrm{mg} / \mathrm{ml}$ & $3 B$ & $14.90 \pm 0.72$ \\
\hline \multirow{3}{*}{ NuoX/NuoY } & FAD ${ }^{2+} 10 \mathrm{mM}$ & $3 \mathrm{~A}$ & $5.03 \pm 1.07$ \\
\cline { 2 - 4 } & Bile salts $10 \mathrm{mg} / \mathrm{ml}$ & $3 B$ & $10.08 \pm 1.37$ \\
\hline
\end{tabular}

Table 2: His-NuoX, His-NuoY and His-NuoX/His-NuoY specific binding fold increase using different substrates.

Proton $\left({ }^{1} \mathrm{H}-\mathrm{NMR}\right)$ and STD-NMR spectra of the His-NuoY fusion protein and of each of the compounds under investigation were acquired as reference spectra; Figure 2 shows the ${ }^{1} \mathrm{H}-\mathrm{NMR}$ spectrum of $\mathrm{FAD}^{2+}$; these spectra served as controls to ensure that any signals observed in ligand: His-NuoY STD difference spectra resulted solely from ligand binding to the protein. The STD-NMR spectrum of $\mathrm{FAD}^{2+}$ interactions with NuoY is shown in Figure 2. ${ }^{1} \mathrm{H}$-NMR spectra of the other compounds and STD-NMR spectra of their interactions with His-NuoY are given in Figure S1.

Binding experiments of $\mathrm{FAD}^{2+}, \mathrm{NAD}^{+}$, fumarate, malate or lactate to NuoY were conducted with 200 equivalents of compound. The STD spectrum of NuoY and $\mathrm{FAD}^{2+}$ clearly showed ligand protons interacting with the NuoY protein (Figure 2 and Figure S1, b). Interestingly, the relative intensities of the resonances in the offresonance ${ }^{1} \mathrm{H}$-NMR spectrum of $\mathrm{FAD}^{2+}$ were not the same as the relative intensities of the signals in the STD-NMR spectrum suggesting that NuoY interacted with different affinities with various moieties of the $\mathrm{FAD}^{2+}$ molecule. This is most evident in the aromatic resonances of the flavin ring at $7.25 \mathrm{ppm}$ that experienced greater reduction of intensity ( $46 \%$ intensity reduction relative to the free flavin methyl resonances) than the aromatic resonances of the adenine moiety at $7.55 \mathrm{ppm}$ and $8.05 \mathrm{ppm}$ (55\% and $75 \%$ reduction, respectively), suggesting a stronger interaction with NuoY for the flavin moiety of $\mathrm{FAD}^{2+}$ than for the adenine moiety.

The STD spectrum of NuoY and $\mathrm{NAD}^{+}$clearly showed NAD+ protons interacting with NuoY (Figure S1, c). Both the adenine and nicotinamide rings of NAD+ appeared to interact with NuoY since their signals are present in the STD difference spectrum. Similarly to the case of $\mathrm{FAD}^{2+}$, the relative intensities of the resonances in the offresonance spectrum were not the same as the signal intensities in the STD spectrum suggesting that NuoY interacted with different affinities with various moieties of the $\mathrm{NAD}^{+}$molecule.

The STD spectrum of NuoY and malate showed the methylene protons interacting with NuoY (Figure S1, e), and similar results were observed for the methine protons of fumarate (Figure S1, d). The STD spectrum of NuoY and lactate however showed no signals attributable to lactate (Figure $\mathrm{S} 1, \mathrm{f}$ ).

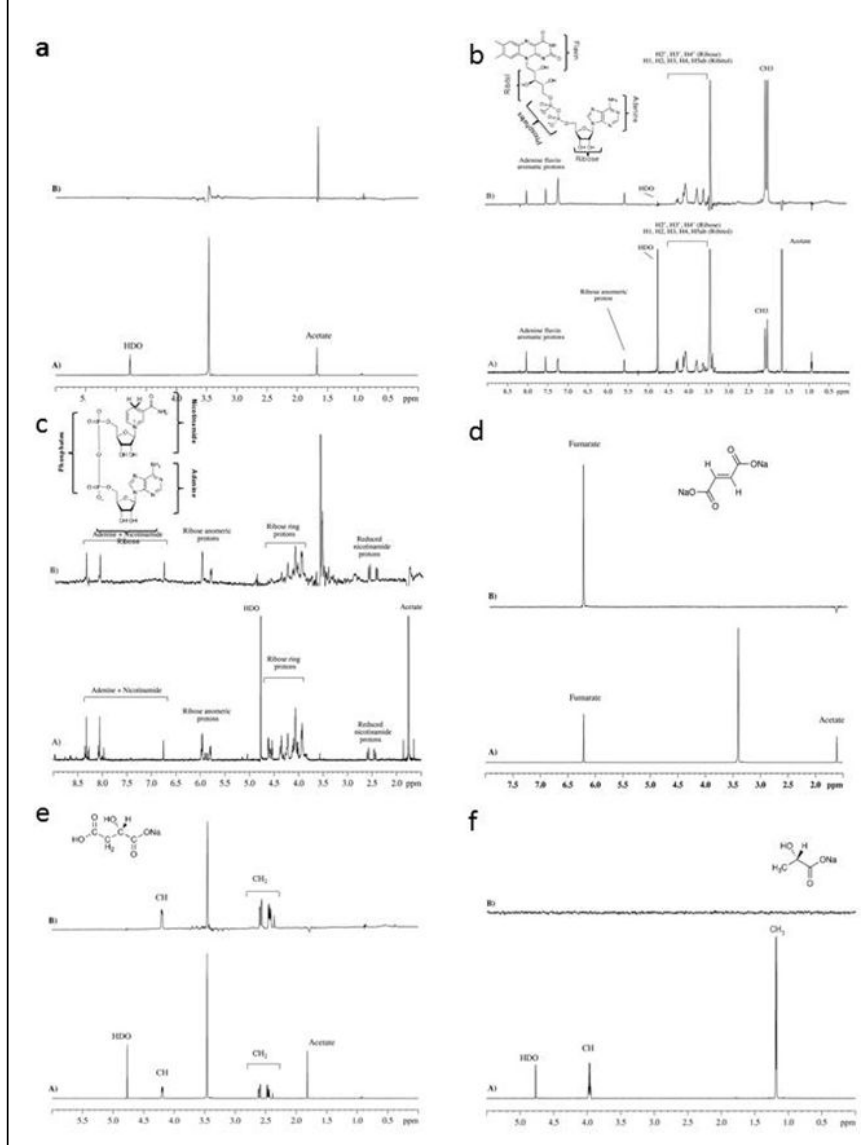

Figure 2: NMR spectra were acquired at $600 \mathrm{MHz}$ and $288 \mathrm{~K}$. Top spectrum: ${ }^{1} \mathrm{H}-\mathrm{NMR}$ of $\mathrm{FAD}^{2+}$. The insert shows a sketch of the $\mathrm{FAD}^{2+}$ molecule. The resonances arising from aromatic flavin and adenine protons, and from ribitol and ribose alipahtic protons are labeled on the spectrum. Bottom spectrum: STD-NMR spectrum of $\mathrm{FAD}^{2+}$ and NuoY protein. The assignment of resonances is the same as in top spectrum.

\section{Characterization of binding affinities of NuoX and NuoY to nucleotide metabolites by SPR}

Surface Plasmon Resonance was employed to determine binding affinities of nucleotide metabolites for NuoX and NuoY at a concentration of $100 \mu \mathrm{g} / \mu \mathrm{l}$ (Table 3). Since in the TCA array assays the NuoX and NuoY proteins bound bile salts, these were used as a trial control analyte for SPR experiments (Table 3). Bile salts are mixed analytes, and they may be used as controls since affinities observed for interactions with them by SPR will be lower than any interactions for single analytes that bind specifically with the proteins. Bile salts had a dissociation constant $\left(K_{\mathrm{D}}\right)$ of $14.8 \mathrm{mM}$ and $4.8 \mathrm{mM}$ for NuoX and NuoY, respectively.

Using SPR, the affinities of $\mathrm{NAD}^{+}, \mathrm{NADH}$ and $\mathrm{FAD}^{2+}$ at concentrations from $10 \mathrm{nM}$ to $1000 \mu \mathrm{M}$ were measured. Lactate and malate binding to NuoX or NuoY could not be analyzed by SPR, because their small molecular weight required concentrations that 
produced precipitation immediately after injection, and consequently blocked the chip.

The His-NuoY protein showed the highest affinity to $\mathrm{FAD}^{2+}$ with a $K_{\mathrm{D}}$ of $327 \mathrm{nM}$, and the $K_{\mathrm{D}}$ of His-NuoX for $\mathrm{FAD}^{2+}$ was $13.8 \mu \mathrm{M}$; and affinities for NAD+ of $4.5 \mu \mathrm{M}$ and $1.7 \mu \mathrm{M}$, respectively. Both proteins had a $K_{\mathrm{D}}$ of approximately $430 \mathrm{nM}$ for NADH (Table 3 ).

\section{Discussion}

The study was designed to investigate the interactions and binding capabilities of the $C$. jejuni proteins NuoX and NuoY to various substrates employing a yeast two-hybrid system, a TCA microarray technique, STD-NMR spectroscopy and SPR assays.

\begin{tabular}{|c|c|c|}
\hline Compound & MW & $\mathrm{KD}^{*}$ \\
\hline NuoX & 11,300 & \\
\hline Bile Salts & 430.6 & $14.8 \mathrm{mM} \pm 2.5 \mathrm{mM}$ \\
\hline $\mathrm{FAD}^{2+}$ & 829.51 & $13.8 \mu \mathrm{M} \pm 0.4 \mu \mathrm{M}$ \\
\hline $\mathrm{NAD}^{+}$ & 685.4 & $1.74 \mu \mathrm{M} \pm 0.2 \mu \mathrm{M}$ \\
\hline $\mathrm{NADH}$ & 663.4 & $403 \mathrm{nM} \pm 69 \mathrm{nM}$ \\
\hline NuoY & 26,000 & \\
\hline Bile salts & 430.6 & $4.8 \mathrm{mM} \pm 0.3 \mathrm{mM}$ \\
\hline $\mathrm{FAD}^{2+}$ & 829.51 & $327 \mathrm{nM} \pm 315 \mathrm{nM}$ \\
\hline $\mathrm{NAD}^{+}$ & 685.4 & $4.5 \mu \mathrm{M} \pm 0.4 \mu \mathrm{M}$ \\
\hline $\mathrm{NADH}$ & 663.4 & $478 \mathrm{nM} \pm 55 \mathrm{nM}$ \\
\hline
\end{tabular}

Table 3: Average affinity dissociation (KD) for each compound tested with His-NuoX and His-NuoY by SPR. *Affinity data are shown as the average of a minimum of two repeats with error as the mean of the error of all measurements.

It has been hypothesized that in Complex I, these two Nuo proteins interact with each other and with the adjacent proteins, to transfer signals to the membrane fragment of the Complex. It has been proposed that the gene organization in Complex I operons is related to the spatial arrangement of the subunits [37]. The periplasmic fragment appears to be an interchangeable input module [14], whereas the function of the $\mathrm{N}$-module of the peripheral arm (NuoE, $-\mathrm{F},-\mathrm{G}$ ) is to oxidize NADH and to transfer the electrons to the membrane arm [35]. Yano et al. [19] discussed the possibility that interactions with neighboring subunits, such as the NuoG subunit, may be required for cofactor incorporation and to form a more stable subcomplex [19]. Similar loci of nuo $X$ and nuo $Y$ in the $C$. jejuni nuo operon relative to those of nuoE and nuoF in the E. coli nuo operon [20], suggested that NuoX and NuoY would be located in the peripheral fragment of Complex I like the E. coli enzymes [19]. To determine whether NuoX and NuoY of $C$. jejuni interacted as do those of the NuoE and NuoF of $E$. coli, protein-protein interactions of the $C$. jejuni proteins were investigated in a yeast two-hybrid system, and the results suggested that NuoX and NuoY indeed interacted.

The NuoG of several bacterial species can be segregated to form a soluble unit which possesses NADH dehydrogenase activity [17-20]. To investigate whether $C$. jejuni NuoG may be required to form a more stable functional complex with NuoX/NuoY subcomplex, numerous unsuccessful attempts were made at cloning intermediates for $C$. jejuni NuoG in an $E$. coli host. This could be due to deleterious effects on $E$. coli cells of having two copies of the nuoG gene in the transformed host. Falk-Krzesinski and Wolfe [38,39] demonstrated that if subunit NuoG of E. coli was deleted or duplicated, Complex I was prevented from functioning properly.

Since NuoX and NuoY interacted, it was hypothesized that they could have similar roles as E. coli NuoE and NuoF. Thus, the interactions of the $C$. jejuni proteins with various metabolites, including some involved in the TCA cycle, were investigated. To this end, a TCA array was developed based on a technique previously used to analyze interactions of various proteins with glycans, amino acids and lectins $[29,31,40]$. The TCA array was constructed and verified by hybridization with human glutathione reductase, a well characterized and commercially available protein. The protein was proven to be functional in both binding and enzymatic assays [41]. The TCA array successfully identified known interactions for this protein, specifically with $\mathrm{NAD}^{+}$and $\mathrm{FAD}^{2+}$, showing that it was a viable tool for identification of protein-ligand interactions of proteins catalyzing redox reactions with dinucleotide cofactors. The TCA array also included bile salts as controls owing to previous findings that they are chemoattractants for $C$. jejuni [35].

The TCA array assay showed that $\mathrm{FAD}^{2+}$ bound both NuoY and NuoX, but $\mathrm{NAD}^{+}$did not bind either protein (Table 2). NuoY also bound to malate, which can function as both an electron acceptor or donor, and to lactate that can donate protons in the absence of oxygen [26]. Interestingly, more interactions were observed by NMR spectroscopy and SPR analyses as discussed below, indicating that the array did not identify all binding partners. Possibly, this is due to the affinity/kinetics of the interactions, and/or to the orientation of the compounds bound to the array in a way that may obscure the portion of the molecule responsible for binding to NuoY.

The unique combination of the newly designed TCA array and STD-NMR spectroscopy data indicated that NuoY interacts with $\mathrm{FAD}^{2+}$, and the NMR data that it interacted with NAD+. SPR analyses confirmed high affinity of this protein for $\mathrm{FAD}^{2+}, \mathrm{NAD}^{+}$, and NADH. These results suggested that NuoY could bind the flavin adenine dinucleotide and as such, it could mediate electron flow into the respiratory chain from the reduced form of the dinucleotide. The SPR data indicated that both NuoX and NuoY are capable of binding $\mathrm{NADH}$ (with an affinity about ten-fold greater than $\mathrm{NAD}^{+}$for NuoY (Table 3). The results indicated that and that $\mathrm{FAD}^{2+}$ had the highest affinity of all the dinucleotides tested for NuoY (Table 3). If the affinities for $\mathrm{FAD}^{2+}$ and $\mathrm{FADH}_{2}$ for NuoY were in a comparable ratio as those for $\mathrm{NAD}^{+}$and $\mathrm{NADH}$, in vivo $\mathrm{FADH}_{2}$ could be a substrate for NuoY preferred over $\mathrm{NADH}$, with a mid nanomolar affinity. Unfortunately $\mathrm{FADH}_{2}$ is not commercially available and therefore it could not be tested. The binding of NuoY to potential proton donors, such as malate and lactate, observed on the TCA array (Table 2) would support a dehydrogenase role for this enzyme.

In the TCA array NuoX bound only to $\mathrm{FAD}^{2+}$ (Table 2), but showed more diverse interactions in the SPR results (Table 3), similar to those observed for NuoY. The fact that both NuoX and NuoY can bind $\mathrm{NADH}$ and $\mathrm{NAD}^{+}$, raises the possibility that $C$. jejuni may use this to its advantage by scavenging this metabolite from host cells during infection.

This study provided evidence that $C$. jejuni NuoX and NuoY could have similar roles as E. coli NuoE and NuoF of supplying electrons for 
the respiratory chain. The results supported the hypothesis that $\mathrm{FADH}_{2}$ could be a primary source of electrons in $C$. jejuni respiration. The essential nature of NuoX and NuoY for $C$. jejuni growth and proliferation [23] together with the results of this work could be interpreted as these enzymes are essential components in the respiratory chain of $C$. jejuni such that their inhibition would interrupt the electron flow in the respiratory chain of the bacterium with bactericidal effects. The data from this investigative combination of TCR array, SPR and NMR technologies also contributed to the understanding of $C$. jejuni requirement of a microearobic environment to grow.

\section{Author Contributions}

LC-G, CD, LH-T, JW and VK Performed experiments and analyzed results; VK, $\mathrm{CD}$ and GM designed the study and planned experiments; LC-G, GM and VK prepared the manuscript.

\section{Acknowledgement}

Lirio I. Calderon-Gomez was partially supported by Australian Research Council Discovery Project grant.

\section{Conflict of Interest Statement}

Authors do not have any financial or commercial conflicts of interest.

\section{References}

1. Krieg NR, Hoffman PS (1986) Microaerophily and oxygen toxicity. Annu Rev Microbiol 40: 107-130.

2. Parkhill J, Wren BW, Mungall K, Ketley JM, Churcher C, et al. (2000) The genome sequence of the food-borne pathogen Campylobacter jejuni reveals hypervariable sequences. Nature 403: 665-668.

3. Westfall HN, Rollins DM, Weiss E (1986) Substrate utilization by Campylobacter jejuni and Campylobacter coli. Appl Environ Microbiol 52: 700-705.

4. Mendz GL, Ball GE, Meek DJ (1997) Pyruvate metabolism in Campylobacter spp. Biochim Biophys Acta 1334: 291-302.

5. Mohammed KA, Miles RJ, Halablab MA (2004) The pattern and kinetics of substrate metabolism of Campylobacter jejuni and Campylobacter coli. Lett Appl Microbiol 39: 261-266.

6. Velayudhan J, Jones MA, Barrow PA, Kelly DJ (2004) L-serine catabolism via an oxygen-labile L-serine dehydratase is essential for colonization of the avian gut by Campylobacter jejuni. Infect Immun 72: 260-268.

7. Fredrick KL, Helmann JD (1994) Dual chemotaxis signaling pathways in Bacillus subtilis: a sigma D-dependent gene encodes a novel protein with both CheW and CheY homologous domains. J Bacteriol 176: 2727-2735.

8. Finel M (1998) Does NADH play a central role in energy metabolism in Helicobacter pylor? Trends in Biochem Sci 23: 412-414.

9. Yagi T (1986) Purification and characterization of NADH dehydrogenase complex from Paracoccus denitrificans. Arch Biochem Biophys 250: 302-311.

10. Friedrich T (2001) Complex I: a chimaera of a redox and conformationdriven proton pump? J Bioenerg Biomemb 33: 169-177.

11. Weidner U, Geier S, Ptock A, Friedrich T, Leif H, et al. (1993) The Gene Locus of the Proton-translocating NADH: Ubiquinone Oxidoreductase in Escherichia coli: Organization of the 14 Genes and Relationship Between the Derived Proteins and Subunits of Mitochondrial Complex. I J Mol Biol 233: 109-122.

12. Friedrich T, Böttcher B (2004) The gross structure of the respiratory complex I: a Lego System. Biochim Biophys Acta 1608: 1-9.
13. Holt PE (1981) Role of campylobacter spp. in human and animal disease: a review. J R Soc Med 74: 437-440.

14. Dupuis A, Darrouzet E, Duborjal H, Pierrard B, Chevallet M, et al. (1998) Distal genes of the nuo operon of Rhodobacter capsulatus equivalent to the mitochondrial ND subunits are all essential for the biogenesis of the respiratory $\mathrm{NADH}-$ ubiquinone oxidoreductase. Mol Microbiol 28: 531-541.

15. Dupuis A, Prieur I, Lunardi J (2001) Toward a characterization of the connecting module of complex I. J Bioenerg Biomembr 33: 159-168.

16. Yagi T, Yano T, Di Bernardo S, Matsuno-Yagi A (1998) Procaryotic complex I (NDH-1), an overview. Biochim Biophys Acta 1364: 125-133.

17. Leif H, Sled VD, Ohnishi T, Weiss H, Friedrich T (1995) Isolation and characterization of the proton-translocating NADH: ubiquinone oxidoreductase from Escherichia coli. Eur J Biochem 230: 538-548.

18. Herter SM, Schiltz E, Drews G (1997) Protein and gene structure of the NADH-binding fragment of Rhodobacter capsulatus NADH:ubiquinone oxidoreductase. Eur J Biochem 246: 800-808.

19. Yano $T$, Sled VD, Ohnishi T, Yagi $T$ (1996) Expression and characterization of the flavoprotein subcomplex composed of $50-\mathrm{kDa}$ (NQO1) and $25-\mathrm{kDa}$ (NQO2) subunits of the proton-translocating NADH-quinone oxidoreductase of Paracoccus denitrificans. J Biol Chem 271: 5907-5913.

20. Smith MA, Finel M, Korolik V, Mendz GL (2000) Characteristics of the aerobic respiratory chains of the microaerophiles Campylobacter jejuni and Helicobacter pylori. Arch Microbiol 174: 1-10.

21. Hoffman PS, Goodman TG (1982) Respiratory physiology and energy conservation efficiency of Campylobacter jejuni. J Bacteriol 150: 319-326.

22. Weerakoon DR, Olson JW (2008) The Campylobacter jejuni NADH:ubiquinone oxidoreductase (complex I) utilizes flavodoxin rather than NADH. J Bacteriol 190: 915-925.

23. Hughes NJ, Chalk PA, Clayton CL, Kelly DJ (1995) Identification of carboxylation enzymes and characterization of a novel four-subunit pyruvate:flavodoxin oxidoreductase from Helicobacter pylori. J Bacteriol 177: 3953-3959.

24. Pieulle L, Charon MH, Bianco P, Bonicel J, Pétillot Y, et al. (1999) Structural and kinetic studies of the pyruvate-ferredoxin oxidoreductase/ ferredoxin complex from Desulfovibrio africanus. Eur J Biochem 264: 500-508.

25. Kelly DJ (2001) The physiology and metabolism of Campylobacter jejuni and Helicobacter pylori. Symp Ser Soc Appl Microbiol 30: 16S-24S.

26. Weingarten RA, Taveirne ME, Olson JW (2009) The dual-functioning fumarate reductase is the sole succinate:quinone reductase in Campylobacter jejuni and is required for full host colonization. J Bacteriol 191: 5293-5300.

27. Carlone GM, Lascelles J (1982) Aerobic and anaerobic respiratory systems in Campylobacter fetus subsp. jejuni grown in atmospheres containing hydrogen. J Bacteriol 152: 306-314.

28. Liu YM, Denkmann K, Kosciow K, Dahl C, Kelly DJ (2013) Tetrathionate stimulated growth of Campylobacter jejuni identifies a new type of bifunctional tetrathionate reductase (TsdA) that is widely distributed in bacteria. Mol Microbiol 88: 173-88.

29. Hartley-Tassell LE, Shewell LK, Day CJ, Wilson JC, Sandhu R, et al. (2010) Identification and characterization of the aspartate chemosensory receptor of Campylobacter jejuni. Mol Microbiol 75: 710-730.

30. Quevillon-Cheruel S, Collinet B, Trésaugues L, Minard P, Henckes G, et al. (2006) Cloning, Production and Purification of Proteins for a medium-scale Structural Genomics Project. In: Macromolecular Crystallography Protocols, 363: 21-37.

31. Day CJ, Tiralongo J, Hartnell RD, Logue CA, Wilson JC, et al. (2009) Differential carbohydrate recognition by Campylobacter jejuni strain 11168: influences of temperature and growth conditions. PLoS One 4: e4927.

32. Krauth-Siege RL, Comini MA (2008) Redox control in trypanosomatids, parasitic protozoa with trypanothione-based thiol metabolism. Biochim Biophys Acta 1780: 1236-1248. 
Citation: Calderon-Gomez LI, Day CJ, Hartley-Tassell LE, Wilson JC, Mendz GL, et al. (2017) Identification of NuoX and NuoY Ligand Binding Specificity in the Campylobacter Jejuni Complex I. J Bacteriol Parasitol 8: 307. doi:10.4172/2155-9597.1000307

Page 8 of 8

33. Kanzok SM, Fechner A, Bauer H, Ulschmid JK, Müller HM, et al. (2001) Substitution of the thioredoxin system for glutathione reductase in Drosophila melanogaster. Science 291: 643-646.

34. Marchler-Bauer A, Bryant SH (2004) CD-Search: protein domain annotations on the fly. Nucleic Acids Res 32: W327-331.

35. Hugdahl MB, Beery JT, Doyle MP (1988) Chemotactic behavior of Campylobacter jejuni. Infect Immun 56: 1560-1566.

36. Mayer M, Meyer B (1999) Characterization of ligand binding by saturation transfer difference NMR spectroscopy. Angewandte ChemieInt Ed 38: 1784-1788.

37. Di Bernardo S, Yagi T (2001) Direct interaction between a membrane domain subunit and a connector subunit in the $\mathrm{H}(+)$-translocating NADH-quinone oxidoreductase. FEBS Lett 508: 385-388.
38. Guenebaut V, Schlitt A, Weiss H, Leonard K, Friedrich T (1998) Consistent structure between bacterial and mitochondrial NADH:ubiquinone oxidoreductase (complex I). J Mol Biol 276: 105-112.

39. Falk-Krzesinski HJ, Wolfe AJ (1998) Genetic analysis of the nuo locus, which encodes the proton-translocating NADH dehydrogenase in Escherichia coli. J Bacteriol 180: 1174-1184.

40. Arndt NX, Tiralongo J, Madge PD, von Itzstein M, Day CJ (2011) Differential carbohydrate binding and cell surface glycosylation of human cancer cell lines. J Cell Biochem 112: 2230-2240.

41. Smith IK, Vierheller TL, Thorne CA (1988) Assay of glutathione reductase in crude tissue homogenates using 5,5'-dithiobis(2nitrobenzoic acid). Anal Biochem 175: 408-413. 